\title{
MDR1 Polymorphisms and Modulation of ARV Drug Induced Hepatotoxicity
}

HARI OM SINGH ( $\nabla$ hariomsgpgims@gmail.com )

National AIDS Research Institute

Dharmesh Samani

National AIDS Research Institute

\section{Research Article}

Keywords: ARV drug induced hepatotoxicity, Genetic predisposition, NNRTI regimen, Multidrug-resistant protein-1, HIV Infected Individuals

Posted Date: November 8th, 2021

DOI: https://doi.org/10.21203/rs.3.rs-1019782/v1

License: (1) This work is licensed under a Creative Commons Attribution 4.0 International License.

Read Full License 


\section{Abstract \\ Background}

Antiretroviral (ARV) regimen is used to manage the progression of HIV infection. MDR1 Polymorphism is associated with the treatment outcome. Ethnic disparities have been observed in the distribution of MDR1 genotypes. Hence we analyzed the association of MDR1 polymorphism with the modulation of ARV associated hepatotoxicity.MDR1 polymorphisms (1236 C/T, $3435 \mathrm{C} / \mathrm{T})$ was genotyped in a total $165 \mathrm{HIV}$ infected individuals (34 ARV drug induced hepatotoxicity were labeled as cases, 131 those without hepatotoxicity were controls) and 155 healthy individuals by utilization of PCR-RFLP.

\section{Results}

Individuals with haplotype TC of $M D R 1$ were at greater risk for hepatotoxicity severity $(\mathrm{OR}=1.96, \mathrm{P}=0.06)$. Haplotypes TT and CC were associated with a reduced risk of hepatotoxicity severity $(O R=0.16, P=0.006$; $\mathrm{OR}=0.46, \mathrm{P}=0.06$ ). MDR1 1236TT genotype was seen higher among ARV drug induced hepatotoxicity cases who consumed alcohol than non-users $(28.6 \%$ versus $14.8 \%$, OR=1.50). There was an increased prevalence of MDR1 1236TT genotype in ARV drug induced hepatotoxicity cases on nevirapine in contrast with efavirenz (21.7\% versus $9.1 \%$, OR=2.11). The incidence of MDR1 1236CT, 1236TT genotypes was found to be more in individuals those without hepatotoxicity on nevirapine as compared to efavirenz ( $43.7 \%$ versus $33.3 \%, \mathrm{OR}=1.66 ; 12.6 \%$ versus $8.3 \%$, $\mathrm{OR}=1.96)$. MDR1 1236TT genotype in presence of nevirapine and who consumed alcohol showed risk for severity of hepatotoxicity $(40.0 \%$ versus $16.67 \%$, OR=2.21).

\section{Conclusion}

Haplotype TC was associated with hepatotoxicity severity. MDR1 1236TT and 3435CT genotypes in presence of nevirapine and who consume alcohol showed higher risk for acquisition and severity of ARV associated hepatotoxicity.

\subsection{Background}

Antiretroviral (ARV) therapy is being extensively utilized for the management of HIV patients. The death rate among individuals with HIV is constantly increasing. Long-term efficacy and toxicity are the significant issues associated with ARV regimen, to worry about. Hepatotoxicity is an adverse outcome of ARV drugs in HIV-infected individuals [1, 2]. A higher occurrence of hepatotoxicity was seen with the utilization of nevirapine-based regimen than the efavirenz-based regimen [3]. The occurrence of ARV drug induced hepatotoxicity because of nevirapine was shown to be $3.19 \%$ [4]. In another investigation, the occurrence of grade 3 or 4 hepatotoxicity was $10.8 \%$ in the patients treated with efavirenz and $8.9 \%$ in 
patients treated with nevirapine (5). $A B C B 1$ is one of the universal adenosine triphosphate (ATP)-binding cassette $(A B C)$ genes, responsible for cell homeostasis [6-8].

$A B C B 1$ is situated on chromosome $7 q 21$. It is a part of the MDR subfamily [8]. ABCB1 is expressed in a few tissues including epithelial cells of the blood-brain barrier $[9,10]$ and transports numerous drugs [11]. P-glycoprotein (P-gp), a transmembrane transporter protein, is encoded by the $A B C B 1 / M D R 1$ gene. P-gp is an ATP dependent efflux system that transports substances including drugs from intracellular to the extracellular matrix [12-14]. The variation in P-gp expression may vary its function, thus can affect the transport of drugs including nevirapine (NVP) [15]. The absorption and penetration of efavirenz (EFV) and NVP have evidently shown to be influenced by the P-gp expression [16]. Chelule et al., (2003) have reported the prevalence of MDR1 3435CC genotype to be $85.9 \%$ in Africans, $41.70 \%$ in Indians, and $35.7 \%$ in the whites of Kwazulu-Natal and South Africa, respectively. In the African population, the presence of the MDR1 3435CC genotype was found to be associated with an overexpression of P-glycoprotein, whereas, the patients with TT genotype demonstrated a lower expression of P-gp $[17,18]$. Studies have reported significantly increased CD4 cell counts among HIV patients with MDR1 3435TT genotype [19, 20]. Haas et al., (2006) proposed that MDR1 3435C/T polymorphism was not linked to efavirenz exposure [19]. Salem et al., (2014) suggested that MDR1 3435C/T polymorphism had no impact on efavirenz clearance [21]. Zhu et al., (2013) proposed that polymorphisms of MDR1 3435T/C and 2677T/G were linked to the response of nevirapine treatment $(P=0.031, P=0.001)$ and could help to predict the drug response in HIV patients [22]. MDR1 3435TT genotype among individuals treated with EFV or nelfinavir correlated with a more elevated level of CD4+ count than the CT/CC genotypes [23]. Leschziner et al., (2007) showed that MDR1 3435 TT genotype were linked with higher adverse outcomes of 3TC (Lamivudine) and NVP treatment than EFV treatment [24]. Ritchie et al. (2006) indicated a significantly reduced risk of hepatotoxicity with NNRTI-containing regimens in the presence of MDR1 3435C/T polymorphism [25]. The link between polymorphism of MDR1 3435C/T and nevirapine induced hepatotoxicity has also been documented by studies $[25,26]$.

Few studies have shown an association between MDR1 polymorphism and adverse outcomes of ARV drugs whereas, other studies suggest no correlation. The results were not conclusive. Moreover, the association between MDR1 polymorphism and ARV drug induced hepatotoxicity has not been reported from India. Thus, we have analyzed the association between MDR1 (1236C/T and 3435C/T) polymorphisms and modulation of ARV drug induced hepatotoxicity.

\subsection{Methods}

\subsection{Study design and population}

The study was carried out at the National AIDS Research Institute (NARI), Pune. The institute harbors an ARV therapy clinic, approved in 2005, by the National AIDS Control Organization (NACO) and provides free HIV testing and antiretroviral drugs under full NACO ARV therapy roll out programme. This works in collaboration with the Pune Municipal Corporation (PMC). Thus the patients are from the PMC area. A 
case control study was conducted. The study population consisted of a cohort of patients who tested HIV positive and were on ARV therapy from August 2014 to September 2017, in the NARI clinic. Patients start ARV therapy based on the WHO criteria for the ARV therapy initiation in the adults and adolescents. All the individuals in this study were on first line ARV regimen, NNRTIs (Non-nucleoside reverse transcriptase inhibitors) during the period of study. A subclassification of the study population was made on the basis of liver function test (LFT) and this subset of population was defined as the HIV-infected individuals with ARV drug induced hepatotoxicity were labeled as cases. The male patients with SGOT $>93.8 \mathrm{U} / \mathrm{ml}$, Alkaline phosphatase $>550.8 \mathrm{U} / \mathrm{ml}$, total bilirubin $>3.22 \mathrm{mg} / \mathrm{ml}$ and SGPT $>229.5 \mathrm{U} / \mathrm{ml}$ and female patients with SGOT $>163.2 \mathrm{U} / \mathrm{ml}$, Alkaline phosphatase $>550.8 \mathrm{U} / \mathrm{ml}$, total bilirubin $>3.22 \mathrm{mg} / \mathrm{ml}$ and SGPT>173.4 U/ml were classified as case group possessing hepatotoxicity. Exclusion criteria: (1) patients with Tuberculosis, Hepatitis B and C infections (2) patients with immune reconstitution syndrome and untreated opportunistic infections (3) patients on other known hepatotoxic medications for the case group showing hepatotoxicity.

At the same time HIV-infected individuals those without hepatotoxicity were labeled as a control group was age matched and recruited. Control population for the HIV positive group, tested negative for TB, Hepatitis B and C and also was not from a similar family as the study cases. The HIV infected males and females with, SGOT $<32 \mathrm{U} / \mathrm{ml}$, Alkaline phosphatase $<108 \mathrm{U} / \mathrm{ml}$, total bilirubin $<1.24 \mathrm{mg} / \mathrm{ml}$, and $\mathrm{SGPT}<34 \mathrm{U} / \mathrm{ml}$. For the purpose of analysis the independent variables were categorized as: variables related to HIV (CD4+ count to define the stage of HIV infection and NNRTI regimen for drug induced hepatotoxic status), variables related to habits and lifestyle (drinking and tobacco usage) and physiological variables like the status of the liver enzymes.

Clinical information was noted through the reviews of case records, questionnaires, and personal interviews. The ethical endorsement was taken from the Ethics Committee, National AIDS Research Institute, Pune, India (Reference number: August 28, 2013, EC/NARI/Genetic Susceptibility/13-14/146) and written consents were taken from every single qualified subject.

The stages of HIV infection were defined on the basis of CD4+ cell counts of the patients at the time of recruitment. Fluorescence-activated cell sorting (FACS) analysis was utilized to estimate the CD4+ count. CD $4+$ cell counts of $<200$ cells $/ \mathrm{mm} 3,201-350$ cells $/ \mathrm{mm} 3$, and $>350$ cells $/ \mathrm{mm} 3$ were considered as advanced, intermediate and early stages of HIV infection, respectively.

HBsAg and hepatitis C testing was completed by ELISA with the Ortho HCV ELISA test system.

With regard to the ARV therapy, Efavirenz and Nevirapine were the antiretrovirals administered. A questionnaire was utilized to record the usage of tobacco and alcohol.

\subsection{Extraction of DNA}

The collection of $2 \mathrm{ml}$ peripheral blood was done from all subjects and put at $-70^{\circ} \mathrm{C}$ until DNA extraction. The DNA extraction was done from blood leukocytes pellets utilizing the QIAamp DNA Mini Kit according to the kit manual. 


\subsection{Genotyping}

Restriction fragment length polymorphism (RFLP) analysis was utilized to genotype the MDR1 (1236 C/T and $3435 \mathrm{C} / \mathrm{T}$ ) polymorphism. Primers to amplify the MDR1 (C1236T and C3435T) polymorphism were utilized as designated by the previous report [27]. PCR was performed in a volume of $20 \mu \mathrm{l}$. The PCR conditions for amplification of MDR 1 C1236T and C3435T polymorphisms were used as described previously [28]. A thermocycler was utilized to complete all the reactions. PCR products were visualized by ethidium bromide staining. The PCR products of MDR1 C1236T and C3435T polymorphism was digested by utilization of restriction enzymes Haelll and $\mathrm{Mbol}$ at $37^{\circ} \mathrm{C}$ for 16 hours separately. $10 \%$ polyacrylamide gel along with molecular weight markers was utilized to the genotype of MDR1 C1236T and C3435T polymorphisms. The sequences and location of SNPs were employed for genotyping of MDR1 C1236T and C3535T polymorphisms. Genotypes for MDR1 C1236T were: 93bp and 87bp for CC, 87, 58, and 35bp for TT, and 93, 87, 58, and 35bp for CT; for MDR1 C3435T: 130 and 76bp for CC, 206bp for TT and 206, 130, and 76bp for CT. Re-genotyping of $20 \%$ of the samples were done to check the disparities in genotyping by addi1tional staff. The errors in genotyping were cross-verified by DNA sequencing of $10 \%$ of the samples.

\subsection{Statistical examination}

The variable, age was communicated as mean \pm standard deviation (SD). Hardy-Weinberg equilibrium was examined by utilization of the Chi-Square goodness of fit test in healthy individuals. Fisher's exact test was utilized to analyze the genotype frequencies between groups. Logistic regression was utilized to compute the odds ratios (ORs) and 95\% confidence interval (CI). SPSS (SPSS Inc) programming form 17.0 was utilized for statistical examination and the two-sided value was taken as a test of statistical significance. A $p$-value under $\leq 0.05$ was considered for significance. SNPStats online software was utilized to compare the frequency of haplotypes among groups (26). Linkage disequilibrium (LD) was analyzed between both the loci by computing the relative LD value ( $\left.D^{\prime}\right)$ as $D^{\prime}=$ Dij/ Dmax (28). The Dij value was compared among various groups by the comparison of confidence intervals.

\subsection{Results}

Out of who tested HIV positive and were on ARV therapy from August 2014 to September 2017, in the NARI clinic. A total of 165 HIV positive individuals, on ARV therapy who visited NARI clinic from August 2014 to September 2017 were considered for the study. Out of these, 34 individuals showed ARV drug induced hepatotoxicity and constituted the first case group of HIV positive individuals. The remaining 131 individuals didn't show hepatotoxicity (by LFT) and formed the second study group of HIV positive individuals on ARV therapy without ARV drug induced hepatotoxicity. Control population consisted of 155 healthy individuals. The demographic profiles of the participants are outlined in Table 1. 
Table 1

Characteristics of the study populations

\begin{tabular}{|c|c|c|c|}
\hline Subjects & $\begin{array}{l}\text { ARV drug induced } \\
\text { hepatotoxicity }\end{array}$ & $\begin{array}{l}\text { Individuals those without } \\
\text { hepatotoxicity y }\end{array}$ & $\begin{array}{l}\text { Healthy } \\
\text { controls }\end{array}$ \\
\hline Number & $N=34$ & $N=131$ & $N=155$ \\
\hline $\begin{array}{l}\text { Mean Age } \\
\text { (Range) }\end{array}$ & $37.24 \pm 3.29$ & $40.27 \pm 2.45$ & $37.25 \pm 6.30$ \\
\hline Females & 16(47.05) & 44(33.58) & $40(25.80)$ \\
\hline Males & 18(52.94) & $84(64.12)$ & 112(72.25) \\
\hline \multicolumn{4}{|c|}{ NNRTI Regimen } \\
\hline $\begin{array}{l}\text { Efavirenz } \\
\mathrm{N}=23\end{array}$ & 11 (32.35) & $12(9.16)$ & $\begin{array}{l}\text { Not applicable } \\
\text { (NA) }\end{array}$ \\
\hline Nevirapine & 23(67.64) & 119 (90.83) & Not applicable \\
\hline \multicolumn{4}{|l|}{$N=142$} \\
\hline \multicolumn{4}{|l|}{ Alcohol habit } \\
\hline Users $\mathrm{N}=51$ & $7(20.58)$ & 44(33.58) & 0 \\
\hline Non users & 27(79.41) & $87(66.41)$ & 0 \\
\hline \multicolumn{4}{|l|}{$\mathrm{N}=114$} \\
\hline \multicolumn{4}{|c|}{ Tobacco habit } \\
\hline User $N=50$ & $23(67.64)$ & $27(20.61)$ & 0 \\
\hline $\begin{array}{l}\text { Non user } \\
\mathrm{N}=115\end{array}$ & $11(32.35)$ & 104(79.38) & 0 \\
\hline \multicolumn{4}{|l|}{ CD4+ Status } \\
\hline $0-200(\mathrm{~N}=95)$ & $16(16.84)$ & $79(83.16)$ & NA \\
\hline $\begin{array}{l}201- \\
350(N=50)\end{array}$ & $17(50)$ & $33(25.19)$ & NA \\
\hline$>350(\mathrm{~N}=20)$ & $1(2.94)$ & $19(14.50)$ & NA \\
\hline \multicolumn{4}{|c|}{ Abbreviations: NNRTI, Non-nucleoside reverse-transcriptase inhibitors; NA, Not applicable; } \\
\hline $\mathrm{N}$, total numb & subject participant & l and tobacco status was & \\
\hline
\end{tabular}

The mean age \pm SD of HIV infected individuals those without hepatotoxicity, with ARV drug induced hepatotoxicity and healthy individuals were $40.27 \pm 2.45,37.24 \pm 3.29$, and $37.25 \pm 6.30$ years. Each of the study population and control group has been further characterized on the basis of the NNRTI regimen (Efavirenz or Navirapine), alcohol and tobacco usage (users or non-users) and the CD4+ status (to define 
the stage of HIV infection). These parameters were used to categorize the study populations and the control group and the incidence of MDR1 polymorphisms were analyzed in all the categories as shown in the Tables 6-9. 
Table 6

Distribution of MDR1 (1236 C/T and $3435 \mathrm{C} / \mathrm{T}$ ) genotypes among individuals with ARV drug induced hepatotoxicity and those without hepatotoxicity

\begin{tabular}{|c|c|c|c|c|}
\hline $\begin{array}{l}\text { Genotype MDR1 (1236 } \\
\mathrm{C} / \mathrm{T})\end{array}$ & $\begin{array}{l}\text { Tobacco users } \\
\mathrm{N}=7 \text { (\%) }\end{array}$ & $\begin{array}{l}\text { Tobacco non- } \\
\text { users } \\
\mathrm{N}=27(\%)\end{array}$ & $\begin{array}{l}P \\
\text { Value }\end{array}$ & $\mathrm{OR}(95 \% \mathrm{Cl})$ \\
\hline \multicolumn{5}{|c|}{ Individuals with ARV drug induced hepatotoxicity } \\
\hline $\mathrm{CC}$ & $4(57.1 \%)$ & $12(44.4 \%)$ & - & 1 (Reference) \\
\hline CT & $1(14.3 \%)$ & $11(40.7 \%)$ & 0.30 & $\begin{array}{l}0.28(0.024 \text { - } \\
3.18)\end{array}$ \\
\hline TT & $2(28.6 \%)$ & $4(14.8 \%)$ & 0.88 & $\begin{array}{l}1.50(0.13- \\
17.35)\end{array}$ \\
\hline \multirow[t]{2}{*}{$\begin{array}{l}\text { Genotype MDR1 (3435 } \\
\mathrm{C} / \mathrm{T} \text { ) }\end{array}$} & \multirow[t]{2}{*}{$\begin{array}{l}\text { Tobacco users } \mathrm{N}=7 \\
\text { (\%) }\end{array}$} & $\begin{array}{l}\text { Tobacco non- } \\
\text { users }\end{array}$ & \multirow[t]{2}{*}{$\begin{array}{l}\mathrm{P}- \\
\text { Value }\end{array}$} & \multirow[t]{2}{*}{$\mathrm{OR}(95 \% \mathrm{Cl})$} \\
\hline & & $\mathrm{N}=27(\%)$ & & \\
\hline $\mathrm{CC}$ & $3(42.9 \%)$ & $5(18.5 .0 \%)$ & - & 1 (Reference) \\
\hline CT & $2(28.6 \%)$ & $12(44.4 \%)$ & 0.37 & $\begin{array}{l}0.37(0.042- \\
3.25)\end{array}$ \\
\hline TT & $2(28.6 \%)$ & $10(37.0 \%)$ & 0.59 & $\begin{array}{l}0.53(0.051- \\
5.40)\end{array}$ \\
\hline \multicolumn{5}{|c|}{ Individuals those without hepatotoxicity } \\
\hline \multirow[t]{2}{*}{$\begin{array}{l}\text { Genotype MDR1 (1236 } \\
\mathrm{C} / \mathrm{T})\end{array}$} & Tobacco users & \multirow{2}{*}{$\begin{array}{l}\text { Tobacco non- } \\
\text { user } \\
\mathrm{N}=\mathbf{8 8}(\%)\end{array}$} & \multirow{2}{*}{$\begin{array}{l}\mathrm{P}- \\
\text { Value }\end{array}$} & \multirow[t]{2}{*}{$\mathrm{OR}(95 \% \mathrm{Cl})$} \\
\hline & $\mathrm{N}=43(\%)$ & & & \\
\hline $\mathrm{CC}$ & $19(44.2 \%)$ & $40(45.5 \%)$ & - & 1 (Reference) \\
\hline СT & $19(44.2 \%)$ & $37(42.0 \%)$ & 0.44 & $\begin{array}{l}1.39(0.60- \\
3.20)\end{array}$ \\
\hline TT & $5(11.6 \%)$ & $11(12.5 \%)$ & 0.90 & $\begin{array}{l}1.08(0.31- \\
3.75)\end{array}$ \\
\hline \multirow[t]{2}{*}{$\begin{array}{l}\text { Genotype MDR1 (3435 } \\
\mathrm{C} / \mathrm{T} \text { ) }\end{array}$} & \multirow[t]{2}{*}{$\begin{array}{l}\text { Tobacco users } \mathrm{N}=43 \\
\text { (\%) }\end{array}$} & $\begin{array}{l}\text { Tobacco non- } \\
\text { user }\end{array}$ & \multirow[t]{2}{*}{$\begin{array}{l}\mathrm{P}- \\
\text { Value }\end{array}$} & \multirow[t]{2}{*}{$\mathrm{OR}(95 \% \mathrm{Cl})$} \\
\hline & & $\mathrm{N}=88(\%)$ & & \\
\hline CC & $7(16.3 \%)$ & 17 (19.3\%) & - & 1 (Reference) \\
\hline
\end{tabular}

$\mathrm{N}=$ number of subjects, $(\%)=$ frequency of subjects, OR and $95 \%$ Cls were derived from logistic regression model comparing the homozygous wild-type genotype/allele (CC genotype and C allele for MDR1 $1236 \mathrm{C} / \mathrm{T}$ and $3435 \mathrm{C} / \mathrm{T}$ ) with other genotypes. 


\begin{tabular}{|lllll|}
\hline $\begin{array}{l}\text { Genotype MDR1 (1236 } \\
\text { C/T) }\end{array}$ & $\begin{array}{l}\text { Tobacco users } \\
\text { N= 7 (\%) }\end{array}$ & $\begin{array}{l}\text { Tobacco non- } \\
\text { users } \\
\mathbf{N}=\mathbf{2 7}(\%)\end{array}$ & $\begin{array}{l}P \\
\text { Value }\end{array}$ & OR( 95\%Cl) \\
\hline CT & $17(39.5 \%)$ & $33(37.5 \%)$ & 0.74 & $\begin{array}{l}0.80(0.28- \\
2.46)\end{array}$ \\
\hline TT & $19(44.2 \%)$ & $38(43.2 \%)$ & 0.98 & $\begin{array}{l}1.006(0.43- \\
2.32)\end{array}$ \\
\hline $\begin{array}{l}\text { N= number of subjects, }(\%)=\text { frequency of subjects, OR and 95\% Cls were derived from logistic } \\
\text { regression model comparing the homozygous wild-type genotype/allele (CC genotype and C allele for } \\
\text { MDR1 1236 C/T and 3435 C/T) with other genotypes. }\end{array}$ & \\
\hline
\end{tabular}


Table 7

Distribution of MDR1 (1236 C/T and $3435 \mathrm{C} / \mathrm{T})$ genotypes among individuals with ARV drug induced hepatotoxicity and those without hepatotoxicity

$\begin{array}{lllll}\begin{array}{l}\text { Genotype MDR1 (1236 } \\ \mathrm{C} / \mathrm{T})\end{array} & \begin{array}{l}\text { Alcohol users N= } \\ (\%)\end{array} & \begin{array}{l}\text { Alcohol non-users } \mathrm{N}= \\ 27(\%)\end{array} & \begin{array}{l}P \\ \text { Value }\end{array} & \text { OR( } 95 \% \mathrm{Cl})\end{array}$

Individuals with ARV drug induced hepatotoxicity

\begin{tabular}{lllll} 
CC & $4(57.1 \%)$ & $12(44.4 \%)$ & - & 1 (Reference) \\
\hline CT & $1(14.3 \%)$ & $11(40.7 \%)$ & 0.33 & $\begin{array}{l}0.29(0.025- \\
3.43)\end{array}$ \\
\hline TT & $2(28.6 \%)$ & $4(14.8 \%)$ & 0.88 & $\begin{array}{l}1.50(0.13- \\
17.35)\end{array}$ \\
$\begin{array}{l}\text { Genotype MDR1 (3435 } \\
\text { C/T) }\end{array}$ & $\begin{array}{l}\text { Alcohol users N= } \\
(\%)\end{array}$ & $\begin{array}{l}\text { Alcohol non-users N= } \\
27(\%)\end{array}$ & $\begin{array}{l}\text { P- } \\
\text { Value }\end{array}$ & OR( $95 \% \mathrm{Cl})$ \\
CC & $3(42.9)$ & $5(18.5 \%)$ & - & 1 (Reference) \\
CT & $3(42.9)$ & $11(40.7 \%)$ & 0.63 & $\begin{array}{l}0.60(0.077- \\
4.73)\end{array}$ \\
\hline TT & $1(14.3)$ & $11(40.7 \%)$ & 0.32 & $0.26(0.018-$ \\
\hline
\end{tabular}

Individuals those without hepatotoxicity

\begin{tabular}{|c|c|c|c|c|}
\hline $\begin{array}{l}\text { Genotype MDR1 (1236 } \\
\text { C/T) }\end{array}$ & $\begin{array}{l}\text { Alcohol users } \mathrm{N}= \\
44(\%)\end{array}$ & $\begin{array}{l}\text { Alcohol non-users N= } \\
87(\%)\end{array}$ & $\begin{array}{l}\mathrm{P}- \\
\text { Value }\end{array}$ & $\mathrm{OR}(95 \% \mathrm{Cl})$ \\
\hline CC & $23(52.3 \%)$ & $36(41.4 \%)$ & - & 1(Reference) \\
\hline CT & $18(40.9 \%)$ & 38 (43.7\%) & 0.95 & $\begin{array}{l}1.02(0.45- \\
2.35)\end{array}$ \\
\hline TT & $3(6.8 \%)$ & $13(14.9 \%)$ & 0.20 & $\begin{array}{l}0.40(0.098- \\
1.64)\end{array}$ \\
\hline $\begin{array}{l}\text { Genotype MDR1 (3435 } \\
\text { C/T) }\end{array}$ & $\begin{array}{l}\text { Alcohol users } \mathrm{N}= \\
44(\%)\end{array}$ & $\begin{array}{l}\text { Alcohol non-users } \mathrm{N}= \\
87(\%)\end{array}$ & $\begin{array}{l}\text { P- } \\
\text { Value }\end{array}$ & $\mathrm{OR}(95 \% \mathrm{Cl})$ \\
\hline CC & $6(13.6 \%)$ & $18(20.7 \%)$ & - & 1 (Reference) \\
\hline CT & $22(50.0 \%)$ & $28(32.2 \%)$ & 0.12 & $\begin{array}{l}2.47(0.79- \\
7.70)\end{array}$ \\
\hline TT & $16(36.4 \%)$ & 41 (47.1\%) & 0.81 & $\begin{array}{l}1.15(0.37- \\
3.59)\end{array}$ \\
\hline
\end{tabular}


Table 8

Distribution of MDR1 (1236 C/T and $3435 \mathrm{C} / \mathrm{T}$ ) genotypes among individuals with ARV drug induced hepatotoxicity and those without hepatotoxicity

\begin{tabular}{|c|c|c|c|c|}
\hline \multirow[t]{2}{*}{$\begin{array}{l}\text { Genotype } \\
\text { MDR1 } \\
(1236 \mathrm{C} / \mathrm{T})\end{array}$} & $\begin{array}{l}\text { Individuals those without } \\
\text { hepatotoxicity taking } \\
\text { nevirapine }\end{array}$ & $\begin{array}{l}\text { Individuals those without } \\
\text { hepatotoxicity taking } \\
\text { efavirenz }\end{array}$ & $\begin{array}{l}\mathrm{P}- \\
\text { Value }\end{array}$ & $\mathrm{OR}(95 \% \mathrm{Cl})$ \\
\hline & $\mathrm{N}=142(\%)$ & $\mathrm{N}=23(\%)$ & & \\
\hline $\mathrm{CC}$ & $63(44.4)$ & $12(52.2)$ & 1 & Reference \\
\hline CT & $59(41.5)$ & $9(39.1)$ & 0.56 & $\begin{array}{l}1.33(0.51- \\
3.47)\end{array}$ \\
\hline TT & $20(14.1)$ & $2(8.7)$ & 0.42 & $\begin{array}{l}1.93(0.39- \\
9.45)\end{array}$ \\
\hline $\begin{array}{l}\text { Genotype } \\
\text { MDR1 } \\
\text { (3435 C/T) }\end{array}$ & $\begin{array}{l}\text { Individuals those without } \\
\text { hepatotoxicity taking } \\
\text { nevirapine } \mathrm{N}=142(\%)\end{array}$ & $\begin{array}{l}\text { Individuals those without } \\
\text { hepatotoxicity taking } \\
\text { efavirenz } \mathrm{N}=\mathbf{2 3}(\%)\end{array}$ & $\begin{array}{l}\text { P- } \\
\text { Value }\end{array}$ & OR( $95 \% \mathrm{Cl})$ \\
\hline$-* / s C C$ & $31(21.8)$ & $1(4.3)$ & 1 & Reference \\
\hline CT & $52(36.6)$ & $12(52.2)$ & 0.063 & $\begin{array}{l}0.14(0.017- \\
1.11)\end{array}$ \\
\hline TT & $59(41.5)$ & $10(43.5)$ & 0.14 & $\begin{array}{l}0.20(0.025- \\
1.68)\end{array}$ \\
\hline \multicolumn{5}{|c|}{ Individuals with ARV drug induced hepatotoxicity } \\
\hline \multirow[t]{2}{*}{$\begin{array}{l}\text { Genotype } \\
\text { MDR1 } \\
(1236 \mathrm{C} / \mathrm{T})\end{array}$} & $\begin{array}{l}\text { Individuals with } \\
\text { ARV drug induced } \\
\text { hepatotoxicity taking } \\
\text { Nevirapine }\end{array}$ & $\begin{array}{l}\text { Individuals with } \\
\text { ARV drug induced } \\
\text { hepatotoxicity taking } \\
\text { Efavirenz }\end{array}$ & $\begin{array}{l}\text { P- } \\
\text { Value }\end{array}$ & $\mathrm{OR}(95 \% \mathrm{Cl})$ \\
\hline & $\mathrm{N}=23(\%)$ & $\mathrm{N}=11(\%)$ & & \\
\hline $\mathrm{CC}$ & $11(47.8 \%)$ & $5(45.5 \%)$ & - & 1(Reference) \\
\hline CT & $7(30.4 \%)$ & $5(45.5 \%)$ & 0.64 & $\begin{array}{l}0.69(0.14- \\
3.35)\end{array}$ \\
\hline TT & $5(21.7 \%)$ & $1(9.1 \%)$ & 0.55 & $\begin{array}{l}2.11(0.18 \text { - } \\
24.66)\end{array}$ \\
\hline \multirow[t]{2}{*}{$\begin{array}{l}\text { Genotype } \\
\text { MDR1 } \\
\text { (3435 C/T) }\end{array}$} & $\begin{array}{l}\text { Individuals with } \\
\text { ARV drug induced } \\
\text { hepatotoxicity taking } \\
\text { Nevirapine }\end{array}$ & $\begin{array}{l}\text { Individuals with } \\
\text { ARV drug induced } \\
\text { hepatotoxicity taking } \\
\text { Efavirenz }\end{array}$ & $\begin{array}{l}\text { P- } \\
\text { Value }\end{array}$ & $\mathrm{OR}(95 \% \mathrm{Cl})$ \\
\hline & $\mathrm{N}=23(\%)$ & $\mathrm{N}=11(\%)$ & & \\
\hline
\end{tabular}




\begin{tabular}{|c|c|c|c|c|}
\hline \multicolumn{5}{|c|}{ Individuals those without hepatotoxicity } \\
\hline $\mathrm{CC}$ & $7(30.4 \%)$ & $1(9.1 \%)$ & - & 1(Reference) \\
\hline CT & $8(34.8 \%)$ & $6(54.5 \%)$ & 0.18 & $\begin{array}{l}0.19(0.017- \\
2.11)\end{array}$ \\
\hline TT & $8(34.8 \%)$ & $4(36.4 \%)$ & 0.43 & $\begin{array}{l}0.37(0.030- \\
4.49)\end{array}$ \\
\hline \multicolumn{5}{|c|}{ Individuals those without hepatotoxicity } \\
\hline \multirow[t]{2}{*}{$\begin{array}{l}\text { Genotype } \\
\text { MDR1 } \\
(1236 \mathrm{C} / \mathrm{T})\end{array}$} & \multirow[t]{2}{*}{$\begin{array}{l}\text { Individuals those without } \\
\text { hepatotoxicity taking } \\
\text { Nevirapine } \mathrm{N}=119(\%)\end{array}$} & $\begin{array}{l}\text { Individuals those without } \\
\text { hepatotoxicity taking } \\
\text { Efavirenz }\end{array}$ & \multirow[t]{2}{*}{$\begin{array}{l}\text { P- } \\
\text { Value }\end{array}$} & \multirow[t]{2}{*}{$\mathrm{OR}(95 \% \mathrm{Cl})$} \\
\hline & & $\mathrm{N}=12(\%)$ & & \\
\hline CC & $52(43.7 \%)$ & $7(58.3 \%)$ & - & 1(Reference) \\
\hline CT & $52(43.7 \%)$ & $4(33.3 \%)$ & 0.45 & $\begin{array}{l}1.66(0.44- \\
6.24)\end{array}$ \\
\hline TT & $15(12.6 \%)$ & $1(8.3 \%)$ & 0.55 & $\begin{array}{l}1.96(0.22 \text { - } \\
17.42)\end{array}$ \\
\hline \multirow[t]{2}{*}{$\begin{array}{l}\text { Genotype } \\
\text { MDR1 } \\
(3435 \mathrm{C} / \mathrm{T})\end{array}$} & $\begin{array}{l}\text { Individuals those without } \\
\text { hepatotoxicity taking } \\
\text { Nevirapine }\end{array}$ & $\begin{array}{l}\text { Individuals those without } \\
\text { hepatotoxicity taking } \\
\text { Efavirenz }\end{array}$ & \multirow[t]{2}{*}{$\begin{array}{l}\text { P- } \\
\text { Value }\end{array}$} & \multirow[t]{2}{*}{$\mathrm{OR}(95 \% \mathrm{Cl})$} \\
\hline & $\mathrm{N}=119(\%)$ & $\mathrm{N}=12(\%)$ & & \\
\hline $\mathrm{CC}$ & $24(20.2 \%)$ & $0(0.0 \%)$ & NS & - \\
\hline CT & $44(37.0 \%)$ & $6(50.0 \%)$ & - & 1(Reference) \\
\hline TT & $51(42.9 \%)$ & $6(50.0 \%)$ & 0.81 & $\begin{array}{l}1.16(0.34- \\
3.12)\end{array}$ \\
\hline \multicolumn{5}{|c|}{$\begin{array}{l}\text { NS, not significant. } \mathrm{N}=\text { number of subjects, }(\%)=\text { frequency of subjects, } \mathrm{OR} \text { and } 95 \% \text { Cls were derived } \\
\text { from logistic regression model comparing the homozygous wild-type genotype/allele (CC genotype } \\
\text { and } \mathrm{C} \text { allele for MDR } 1236 \mathrm{C} / \mathrm{T} \text { and } 3435 \mathrm{C} / \mathrm{T}) \text { with other genotypes. }\end{array}$} \\
\hline
\end{tabular}


Table 9

Incidence of MDR1 (1236 C/T and $3435 \mathrm{C} / \mathrm{T})$ genotypes among HIV infected individuals with and without ARV induced hepatotoxicity (subclassified as alcohol and NNRTls users and non-users)

\begin{tabular}{|c|c|c|c|c|}
\hline $\begin{array}{l}\text { Genotype } \\
\text { MDR1 } \\
(1236 \mathrm{C} / \mathrm{T})\end{array}$ & $\begin{array}{l}\text { Alcoholt } \\
\text { Nevirapine users } \\
\mathrm{N}=5(\%)\end{array}$ & $\begin{array}{l}\text { Nevirapine users+ Alcohol } \\
\text { non-user } \\
\mathrm{N}=18(\%)\end{array}$ & $\begin{array}{l}P \\
\text { Value }\end{array}$ & $\mathrm{OR}(95 \% \mathrm{Cl})$ \\
\hline \multicolumn{5}{|c|}{ Individuals with ARV drug induced hepatotoxicity } \\
\hline $\mathrm{CC}$ & $3(60.0 \%)$ & $8(44.44 \%)$ & - & 1(Reference) \\
\hline CT & $0(0.0 \%)$ & $7(38.89 \%)$ & NS & - \\
\hline TT & $2(40.0 \%)$ & $3(16.67 \%)$ & 0.55 & $\begin{array}{l}2.21(0.17- \\
29.21)\end{array}$ \\
\hline $\begin{array}{l}\text { Genotype } \\
\text { MDR1 } \\
(3435 \mathrm{C} / \mathrm{T})\end{array}$ & $\begin{array}{l}\text { Alcoholt } \\
\text { Nevirapine users } \\
\mathrm{N}=5(\%)\end{array}$ & $\begin{array}{l}\text { Nevirapine users+ Alcohol } \\
\text { non-user } \\
\mathrm{N}=18(\%)\end{array}$ & $\begin{array}{l}\mathrm{P}- \\
\text { Value }\end{array}$ & $\mathrm{OR}(95 \% \mathrm{Cl})$ \\
\hline $\mathrm{CC}$ & $3(60.0 \%)$ & $4(22.22 \%)$ & - & 1(Reference) \\
\hline CT & $1(20.0 \%)$ & $7(38.39 \%)$ & 0.38 & $\begin{array}{l}0.27(0.015- \\
5.01)\end{array}$ \\
\hline TT & $1(20.0 \%)$ & $7(38.39 \%)$ & 0.75 & $\begin{array}{l}0.61(0.031- \\
12.05)\end{array}$ \\
\hline $\begin{array}{l}\text { Genotype } \\
\text { MDR1 }\end{array}$ & $\begin{array}{l}\text { Alcohol+ Efavirenz } \\
\text { users }\end{array}$ & $\begin{array}{l}\text { Efavirenz users+ Alcohol non- } \\
\text { users }\end{array}$ & $\begin{array}{l}\text { P- } \\
\text { Value }\end{array}$ & $\mathrm{OR}(95 \% \mathrm{Cl})$ \\
\hline$(1236 \mathrm{C} / \mathrm{T})$ & $\mathrm{N}=2(\%)$ & $\mathrm{N}=9(\%)$ & & \\
\hline $\mathrm{CC}$ & $1(50.0 \%)$ & $4(44.44 \%)$ & - & 1(Reference) \\
\hline CT & $1(50.0 \%)$ & $4(44.44 \%)$ & 0.85 & $\begin{array}{l}0.73(0.028- \\
18.97)\end{array}$ \\
\hline TT & $0(0.0 \%)$ & $1(11.12 \%)$ & NS & - \\
\hline $\begin{array}{l}\text { Genotype } \\
\text { MDR1 }\end{array}$ & $\begin{array}{l}\text { Alcohol+ Efavirenz } \\
\text { users }\end{array}$ & $\begin{array}{l}\text { Efavirenz users+ Alcohol non- } \\
\text { users }\end{array}$ & $\begin{array}{l}\text { P- } \\
\text { Value }\end{array}$ & OR( $95 \% \mathrm{Cl})$ \\
\hline (3435 C/T) & $\mathrm{N}=2(\%)$ & $\mathrm{N}=9(\%)$ & & \\
\hline $\mathrm{CC}$ & $0(0.0 \%)$ & $1(11.12 \%)$ & NS & - \\
\hline CT & $2(100 \%)$ & 4 (44.44\%) & - & 1(Reference) \\
\hline
\end{tabular}

NS, not significant. $\mathrm{N}=$ number of subjects, $(\%)=$ frequency of subjects, OR and $95 \%$ Cls were derived from logistic regression model comparing the homozygous wild-type genotype/allele (CC genotype and $\mathrm{C}$ allele for MDR1 $1236 \mathrm{C} / \mathrm{T}$ and $3435 \mathrm{C} / \mathrm{T}$ ) with other genotypes. 


\begin{tabular}{|c|c|c|c|c|}
\hline $\begin{array}{l}\text { Genotype } \\
\text { MDR1 } \\
(1236 \mathrm{C} / \mathrm{T})\end{array}$ & $\begin{array}{l}\text { Alcohol+ } \\
\text { Nevirapine users } \\
\mathrm{N}=5(\%)\end{array}$ & $\begin{array}{l}\text { Nevirapine users+ Alcohol } \\
\text { non-user } \\
\mathrm{N}=18(\%)\end{array}$ & $\begin{array}{l}P \\
\text { Value }\end{array}$ & $\mathrm{OR}(95 \% \mathrm{Cl})$ \\
\hline TT & $0(0.0 \%)$ & $4(44.44 \%)$ & NS & - \\
\hline \multicolumn{5}{|c|}{ Individuals those without hepatotoxicity } \\
\hline $\begin{array}{l}\text { Genotype } \\
\text { MDR1 }\end{array}$ & $\begin{array}{l}\text { Alcohol+ Nevirapine } \\
\text { users }\end{array}$ & $\begin{array}{l}\text { Nevirapine users +Alcohol } \\
\text { non-users }\end{array}$ & $\begin{array}{l}\mathrm{P}- \\
\text { Value }\end{array}$ & $\mathrm{OR}(95 \% \mathrm{Cl})$ \\
\hline$(1236 \mathrm{C} / \mathrm{T})$ & $\mathrm{N}=38(\%)$ & $\mathrm{N}=81(\%)$ & & \\
\hline $\mathrm{CC}$ & $18(47.37 \%)$ & $34(41.98 \%)$ & - & 1(Reference) \\
\hline СT & $17(44.74 \%)$ & $35(43.20 \%)$ & 0.68 & $\begin{array}{l}1.20(0.50- \\
2.89)\end{array}$ \\
\hline TT & $3(7.89 \%)$ & $12(14.82 \%)$ & 0.40 & $\begin{array}{l}0.54(0.13- \\
2.26)\end{array}$ \\
\hline $\begin{array}{l}\text { Genotype } \\
\text { MDR1 }\end{array}$ & $\begin{array}{l}\text { Alcohol+ Nevirapine } \\
\text { users }\end{array}$ & $\begin{array}{l}\text { Nevirapine users+ Alcohol } \\
\text { non-users }\end{array}$ & $\begin{array}{l}\text { P- } \\
\text { Value }\end{array}$ & $\mathrm{OR}(95 \% \mathrm{Cl})$ \\
\hline (3435 C/T) & $\mathrm{N}=38(\%)$ & $\mathrm{N}=81(\%)$ & & \\
\hline $\mathrm{CC}$ & $6(15.78 \%)$ & $18(22.22 \%)$ & - & 1(Reference) \\
\hline CT & $17(44.74 \%)$ & $27(33.33 \%)$ & 0.23 & $\begin{array}{l}2.04(0.64- \\
6.53)\end{array}$ \\
\hline TT & $15(39.47 \%)$ & $36(44.45 \%)$ & 0.74 & $\begin{array}{l}1.22(0.39- \\
3.84)\end{array}$ \\
\hline $\begin{array}{l}\text { Genotype } \\
\text { MDR1 }\end{array}$ & $\begin{array}{l}\text { Alcohol+ Efavirenz } \\
\text { users }\end{array}$ & $\begin{array}{l}\text { Efavirenz users+ Alcohol non- } \\
\text { user }\end{array}$ & $\begin{array}{l}\text { P- } \\
\text { Value }\end{array}$ & $\mathrm{OR}(95 \% \mathrm{Cl})$ \\
\hline$(1236 \mathrm{C} / \mathrm{T})$ & $\mathrm{N}=6(\%)$ & $N=6(\%)$ & & \\
\hline $\mathrm{CC}$ & $5(83.33 \%)$ & $2(33.33 \%)$ & - & 1(Reference) \\
\hline СT & $1(16.67 \%)$ & $3(50.0 \%)$ & 0.16 & $\begin{array}{l}0.13(0.008- \\
2.18)\end{array}$ \\
\hline TT & $0(0.0 \%)$ & $1(16.67 \%)$ & NS & - \\
\hline $\begin{array}{l}\text { Genotype } \\
\text { MDR1 }\end{array}$ & $\begin{array}{l}\text { Alcohol+ Efavirenz } \\
\text { users }\end{array}$ & $\begin{array}{l}\text { Efavirenz users+ Alcohol non- } \\
\text { user }\end{array}$ & $\begin{array}{l}\text { P- } \\
\text { Value }\end{array}$ & $\mathrm{OR}(95 \% \mathrm{Cl})$ \\
\hline (3435 C/T) & $\mathrm{N}=6(\%)$ & $\mathrm{N}=6(\%)$ & & \\
\hline CC & $0(0.0 \%)$ & 0 & NS & - \\
\hline
\end{tabular}




\begin{tabular}{|c|c|c|c|c|}
\hline $\begin{array}{l}\text { Genotype } \\
\text { MDR1 } \\
(1236 \mathrm{C} / \mathrm{T})\end{array}$ & $\begin{array}{l}\text { Alcohol+ } \\
\text { Nevirapine users } \\
\mathrm{N}=5(\%)\end{array}$ & $\begin{array}{l}\text { Nevirapine users+ Alcohol } \\
\text { non-user } \\
\mathrm{N}=18(\%)\end{array}$ & $\begin{array}{l}P \\
\text { Value }\end{array}$ & $\mathrm{OR}(95 \% \mathrm{Cl})$ \\
\hline CT & 5 (83.33\%) & $1(16.67 \%)$ & - & 1(Reference) \\
\hline TT & $1(16.67 \%)$ & 5 (83.33\%) & 0.04 & $\begin{array}{l}0.04(0.002- \\
0.83)\end{array}$ \\
\hline \multicolumn{5}{|c|}{$\begin{array}{l}\text { NS, not significant. } \mathrm{N}=\text { number of subjects, }(\%)=\text { frequency of subjects, } \mathrm{OR} \text { and } 95 \% \text { Cls were derivec } \\
\text { from logistic regression model comparing the homozygous wild-type genotype/allele (CC genotype } \\
\text { and } \mathrm{C} \text { allele for MDR } 1236 \mathrm{C} / \mathrm{T} \text { and } 3435 \mathrm{C} / \mathrm{T} \text { ) with other genotypes. }\end{array}$} \\
\hline
\end{tabular}

\subsection{MDR1 polymorphisms and ARV drug induced hepatotoxicity and control population}

The incidence of MDR1 polymorphisms in the two study populations is shown in Table 2. MDR1 polymorphisms were not found to be distinct between ARV drug induced hepatotoxicity cases and those without hepatotoxicity. Although, among the ARV drug induced hepatotoxicity cases, the predominance of MDR1 1236TT genotype was more as compared to those without hepatotoxicity $(17.6 \%$ versus $12.2 \%$, $\mathrm{OR}=1.38,95 \% \mathrm{Cl}$ : 0.45-4.12, $\mathrm{P}=0.57)$. Whereas, MDR1 3435TT genotype and T allele were underrepresented in the ARV drug induced hepatotoxicity cases as compared to those without hepatotoxicity $(35.3 \%$ versus $43.5 \%, O R=0.56,95 \% \mathrm{Cl}$ : $0.20-1.59, P=0.28$ and $55.88 \%$ versus $62.59 \%$, $\mathrm{OR}=0.65, \mathrm{P}=0.13)$. Also, MDR1 polymorphisms were not significantly different between ARV drug induced hepatotoxicity and the healthy controls. 
Table 2

Distribution of MDR1 (1236 C/T and $3435 \mathrm{C} / \mathrm{T})$ polymorphisms among individuals with ARV drug induced hepatotoxicity and those without hepatotoxicity

\begin{tabular}{|c|c|c|c|c|}
\hline $\begin{array}{l}\text { Genotype } \\
\text { MDR1 (1236 } \\
\mathrm{C} / \mathrm{T})\end{array}$ & $\begin{array}{l}\text { Individuals with } \\
\text { ARV drug induced } \\
\text { hepatotoxicity } \mathrm{N}=34 \text { (\%) }\end{array}$ & $\begin{array}{l}\text { Individuals without } \\
\text { hepatotoxicity } \mathrm{N}=131 \\
\text { (\%) }\end{array}$ & $\begin{array}{l}P \\
\text { Value }\end{array}$ & $\mathrm{OR}(95 \% \mathrm{Cl})$ \\
\hline CC & 16 (47.1\%) & $59(45.0 \%)$ & & 1(Reference) \\
\hline CT & 12 (35.3\%) & $56(42.7 \%)$ & 0.37 & $\begin{array}{l}0.68(0.29- \\
1.60)\end{array}$ \\
\hline TT & $6(17.6 \%)$ & $16(12.2 \%)$ & 0.57 & $\begin{array}{l}1.37(0.45- \\
4.12)\end{array}$ \\
\hline $\mathrm{CT}+\mathrm{TT}$ & $18(52.94)$ & $72(54.96)$ & 0.63 & $\begin{array}{l}0.82(0.38- \\
1.79)\end{array}$ \\
\hline $\begin{array}{l}\text { MDR1 (1236 } \\
\text { C/T) Allele }\end{array}$ & $\begin{array}{l}\text { Individuals with } \\
\text { ARV drug induced } \\
\text { hepatotoxicity } \mathrm{N}=68(\%)\end{array}$ & $\begin{array}{l}\text { Individuals without } \\
\text { hepatotoxicity N= } 262 \\
\text { (\%) }\end{array}$ & $\begin{array}{l}\text { P- } \\
\text { Value }\end{array}$ & $\mathrm{OR}(95 \% \mathrm{Cl})$ \\
\hline C & $44(64.71 \%)$ & $174(66.41 \%)$ & & 1(Reference) \\
\hline $\mathrm{T}$ & $24(35.29 \%)$ & 88 (33.59\%) & 0.91 & $\begin{array}{l}1.03(0.59- \\
1.82)\end{array}$ \\
\hline \multirow[t]{2}{*}{$\begin{array}{l}\text { Genotype } \\
\text { MDR1 (3435 } \\
\text { C/T) }\end{array}$} & \multirow[t]{2}{*}{$\begin{array}{l}\text { Individuals with } \\
\text { ARV drug induced } \\
\text { hepatotoxicity } \mathrm{N}=\mathbf{3 4}(\%)\end{array}$} & $\begin{array}{l}\text { Individuals without } \\
\text { hepatotoxicity }\end{array}$ & \multirow[t]{2}{*}{$\begin{array}{l}\mathrm{P}- \\
\text { Value }\end{array}$} & \multirow[t]{2}{*}{$\mathrm{OR}(95 \% \mathrm{Cl})$} \\
\hline & & $\mathrm{N}=131(\%)$ & & \\
\hline $\mathrm{CC}$ & $8(23.5 \%)$ & 24 (18.3\%) & - & 1(Reference) \\
\hline CT & $14(41.2 \%)$ & $50(38.2 \%)$ & 0.70 & $\begin{array}{l}0.82(0.30- \\
2.25)\end{array}$ \\
\hline TT & 12 (35.3\%) & 57 (43.5\%) & 0.28 & $\begin{array}{l}0.56(0.20- \\
1.59)\end{array}$ \\
\hline $\mathrm{CT}+\mathrm{TT}$ & $26(74.47 \%)$ & 107(81.67\%) & 0.42 & $\begin{array}{l}0.68(0.27- \\
1.71)\end{array}$ \\
\hline $\begin{array}{l}\text { MDR1 (3435 } \\
\text { C/T) Allele }\end{array}$ & $\begin{array}{l}\text { Individuals with } \\
\text { ARV drug induced } \\
\text { hepatotoxicity } \mathrm{N}=68(\%)\end{array}$ & $\begin{array}{l}\text { Individuals without } \\
\text { hepatotoxicity } \mathrm{N}=262 \\
\text { (\%) }\end{array}$ & $\begin{array}{l}\text { P- } \\
\text { Value }\end{array}$ & $\mathrm{OR}(95 \% \mathrm{Cl})$ \\
\hline $\mathrm{C}$ & $30(44.12 \%)$ & $98(37.40 \%)$ & - & 1(Reference) \\
\hline $\mathrm{T}$ & 38 (55.88\%) & $164(62.59 \%)$ & 0.13 & $\begin{array}{l}0.65(0.37- \\
1.14)\end{array}$ \\
\hline
\end{tabular}

$\mathrm{N}$, total number of individuals with ARV drug induced hepatotoxicity (34), those without hepatotoxicity (131) and healthy controls (155). OR and 95\% Cls were derived from logistic regression model comparing the homozygous wild-type genotype/allele (CC genotype and C allele for MDR1 $1236 \mathrm{C} / \mathrm{T}$ and $3435 \mathrm{C} / \mathrm{T}$ ) with other genotypes/alleles. 


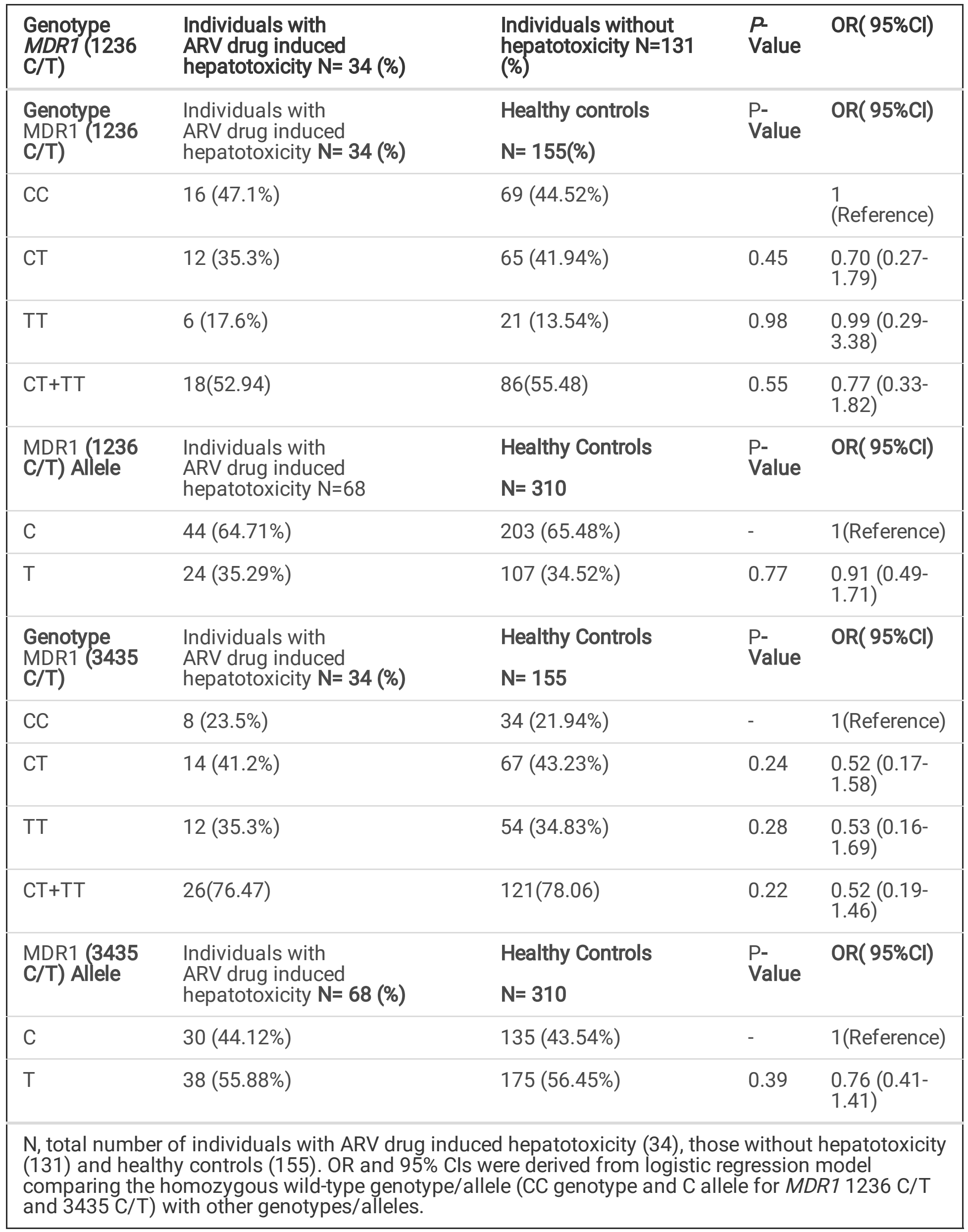




\subsection{MDR1 polymorphism and HIV+ those without ARV drug induced hepatotoxicity}

The occurrence of MDR1 polymorphisms (1236C/T, 3435C/T) in those without hepatotoxicity versus the healthy population is tabulated in Table 3 . The healthy population followed the deviation from HardyWeinberg equilibrium $(P=0.36,0.13)$. The distribution of $M D R 1$ polymorphism was almost alike between those without hepatotoxicity and healthy controls. Although, individuals those without hepatotoxicity had more occurrence of MDR1 3435TT genotype than healthy people (43.5\% versus $34.83 \%, \mathrm{OR}=1.24,95 \% \mathrm{Cl}$ : 0.59-2.61, $\mathrm{P}=0.57)$. The dispersion of other genotypes and alleles of MDR1 polymorphisms were comparable between both groups. 
Table 3

Distribution of MDR1 (1236 C/T and $3435 \mathrm{C} / \mathrm{T})$ polymorphism in individuals those without hepatotoxicity versus healthy controls.

\begin{tabular}{|c|c|c|c|c|}
\hline $\begin{array}{l}\text { Genotype MDR1 } \\
(1236 \mathrm{C} / \mathrm{T})\end{array}$ & $\begin{array}{l}\text { Individuals those without } \\
\text { hepatotoxicity } \mathrm{N}=131(\%)\end{array}$ & $\begin{array}{l}\text { Healthy } \\
\text { controls } \\
\mathrm{N}=155(\%)\end{array}$ & $\begin{array}{l}P \\
\text { Value }\end{array}$ & $\mathrm{OR}(95 \% \mathrm{Cl})$ \\
\hline $\mathrm{CC}$ & $59(45.0 \%)$ & $\begin{array}{l}69 \\
(44.52 \%)\end{array}$ & - & 1 (Reference) \\
\hline CT & $56(42.7 \%)$ & $\begin{array}{l}65 \\
(41.94 \%)\end{array}$ & 0.65 & $\begin{array}{l}0.87(0.48- \\
1.59)\end{array}$ \\
\hline TT & $16(12.2 \%)$ & $\begin{array}{l}21 \\
(13.54 \%)\end{array}$ & 0.39 & $\begin{array}{l}0.69(0.29- \\
1.61)\end{array}$ \\
\hline $\mathrm{CT}+\mathrm{TT}$ & 72(54.96) & $86(55.48)$ & 0.49 & $\begin{array}{l}0.82(0.47- \\
1.43)\end{array}$ \\
\hline \multirow[t]{2}{*}{$\begin{array}{l}\text { MDR1 }(1236 \mathrm{C} / \mathrm{T}) \\
\text { Allele }\end{array}$} & $\begin{array}{l}\text { Individuals those without } \\
\text { hepatotoxicity } \mathrm{N}=262(\%)\end{array}$ & $\begin{array}{l}\text { Healthy } \\
\text { controls }\end{array}$ & $\begin{array}{l}\text { P- } \\
\text { Value }\end{array}$ & $\mathrm{OR}(95 \% \mathrm{Cl})$ \\
\hline & & $N=310$ & & \\
\hline C & $174(66.41 \%)$ & $\begin{array}{l}203 \\
(65.48 \%)\end{array}$ & - & 1 (Reference) \\
\hline $\mathrm{T}$ & 88 (33.59\%) & $\begin{array}{l}107 \\
(34.52 \%)\end{array}$ & 0.37 & $\begin{array}{l}0.83(0.55- \\
1.25)\end{array}$ \\
\hline \multirow[t]{2}{*}{$\begin{array}{l}\text { Genotype MDR1 } \\
(3435 \mathrm{C} / \mathrm{T})\end{array}$} & $\begin{array}{l}\text { Individuals those without } \\
\text { hepatotoxicity } \mathrm{N}=131 \text { (\%) }\end{array}$ & $\begin{array}{l}\text { Healthy } \\
\text { controls }\end{array}$ & $\begin{array}{l}\text { P- } \\
\text { Value }\end{array}$ & OR( $95 \% \mathrm{Cl})$ \\
\hline & & $N=155$ & & \\
\hline $\mathrm{CC}$ & $24(18.3 \%)$ & $\begin{array}{l}34 \\
(21.94 \%)\end{array}$ & - & 1 (Reference) \\
\hline CT & $50(38.2 \%)$ & $\begin{array}{l}67 \\
(43.23 \%)\end{array}$ & 0.53 & $\begin{array}{l}0.79(0.37- \\
1.66)\end{array}$ \\
\hline TT & 57 (43.5\%) & $\begin{array}{l}54 \\
(34.83 \%)\end{array}$ & 0.57 & $\begin{array}{l}1.24(0.59- \\
2.61)\end{array}$ \\
\hline $\mathrm{CT}+\mathrm{TT}$ & 107(81.67) & 121(78.06) & 0.97 & $\begin{array}{l}0.99(0.50- \\
1.94)\end{array}$ \\
\hline \multirow[t]{2}{*}{$\begin{array}{l}\text { MDR1 }(3435 \mathrm{C} / \mathrm{T}) \\
\text { Allele }\end{array}$} & $\begin{array}{l}\text { Individuals those without } \\
\text { hepatotoxicity } \mathrm{N}=\mathbf{2 6 2}(\%)\end{array}$ & $\begin{array}{l}\text { Healthy } \\
\text { controls }\end{array}$ & $\begin{array}{l}\text { P- } \\
\text { Value }\end{array}$ & $\mathrm{OR}(95 \% \mathrm{Cl})$ \\
\hline & & $N=310$ & & \\
\hline
\end{tabular}

$\mathrm{N}$, total number of individuals those without hepatotoxicity (131) and healthy controls (155). OR and $95 \%$ Cls were derived from logistic regression model comparing the homozygous wild-type genotype/allele (CC genotype and C allele for MDR1 $1236 \mathrm{C} / \mathrm{T}$ and $3435 \mathrm{C} / \mathrm{T}$ ) with other genotypes/alleles. 


\begin{tabular}{|c|c|c|c|c|}
\hline $\begin{array}{l}\text { Genotype MDR1 } \\
(1236 \mathrm{C} / \mathrm{T})\end{array}$ & $\begin{array}{l}\text { Individuals those without } \\
\text { hepatotoxicity } \mathrm{N}=131(\%)\end{array}$ & $\begin{array}{l}\text { Healthy } \\
\text { controls } \\
\mathrm{N}=155(\%)\end{array}$ & $\begin{array}{l}P \\
\text { Value }\end{array}$ & $\mathrm{OR}(95 \% \mathrm{Cl})$ \\
\hline C & $98(37.40 \%)$ & $\begin{array}{l}135 \\
(43.54 \%)\end{array}$ & - & 1(Reference) \\
\hline $\mathrm{T}$ & $164(62.59 \%)$ & $\begin{array}{l}175 \\
(56.45 \%)\end{array}$ & 0.41 & $\begin{array}{l}1.18(0.80- \\
1.74)\end{array}$ \\
\hline \multicolumn{5}{|c|}{$\begin{array}{l}\mathrm{N} \text {, total number of individuals those without hepatotoxicity (131) and healthy controls (155). OR and } \\
95 \% \text { Cls were derived from logistic regression model comparing the homozygous wild-type } \\
\text { genotype/allele (CC genotype and C allele for MDR1 } 1236 \mathrm{C} / \mathrm{T} \text { and } 3435 \mathrm{C} / \mathrm{T} \text { ) with other } \\
\text { genotypes/alleles. }\end{array}$} \\
\hline
\end{tabular}

\subsection{Haplotypes distribution}

We have also analyzed the MDR1 haplotypes among the two study groups and the healthy controls, as shown in Table 4. Haplotype CT $\left(1236^{*} \mathrm{C} / 3435^{*} \mathrm{~T}\right)$ was considered as a reference. The incidence of TT haplotype $(1236 * \mathrm{~T} / 3435 * \mathrm{~T})$ has been found to be significantly lesser among ARV drug induced hepatotoxicity cases than those without hepatotoxicity $(0.05 \%$ versus $0.22 \%, \mathrm{OR}=0.16,95 \% \mathrm{Cl}: 0.04-0.059$, $\mathrm{P}=0.0065)$, whereas the incidence of TC haplotype $\left(1236^{*} \mathrm{~T} / 3435^{*} \mathrm{C}\right)$ was significantly more in ARV drug induced hepatotoxicity cases than those without hepatotoxicity $(0.30 \%$ versus $0.11 \%, \mathrm{OR}=1.96,95 \% \mathrm{Cl}$ : 0.98-3.94, $P=0.06$ ). This suggests that haplotype TC was likely to be associated with increased risk for severity of hepatotoxicity because of synergistic effect of gene-gene interaction. The occurrence of CC $\left(1236{ }^{*} \mathrm{C} / 3435^{\star} \mathrm{C}\right)$ and TT $\left(1236^{*} \mathrm{~T} / 3435^{\star} \mathrm{T}\right)$ haplotypes was lesser among ARV drug induced hepatotoxicity cases than healthy controls $(0.14 \%$ versus $0.30 \%, O R=0.34,95 \% \mathrm{Cl}: 0.12-0.94, \mathrm{P}=0.039$, $0.05 \%$ versus $0.22 \%, \mathrm{OR}=0.09,95 \% \mathrm{Cl}: 0.02-0.44, \mathrm{P}=0.0032)$. Whereas, the incidence of TC $\left(1236^{*} \mathrm{~T} / 3435^{\star} \mathrm{C}\right)$ haplotype was predominantly higher in ARV drug induced hepatotoxicity cases compared to the healthy controls $(0.30 \%$ versus $0.13 \%, \mathrm{OR}=1.94,95 \% \mathrm{Cl}: 0.87-4.37, \mathrm{P}=0.11)$. The incidence of MDR1 haplotypes among those without hepatotoxicity individuals was not significantly different from the healthy population. 
Table 4

Distribution of MDR1 haplotypes (1236C/T and 3435C/T) in HIV infected individuals with and without ARV associated hepatotoxicity and healthy controls

\begin{tabular}{|c|c|c|c|c|}
\hline $\begin{array}{l}\text { Haplotype } \\
\text { MDR1 }(1236 \mathrm{C} / \mathrm{T} \\
\text { and } 3435 \mathrm{C} / \mathrm{T})\end{array}$ & $\begin{array}{l}\text { individuals with } \\
\text { ARV drug induced } \\
\text { hepatotoxicity y }(\mathrm{N}=68)\end{array}$ & $\begin{array}{l}\text { individuals } \\
\text { those without } \\
\text { hepatotoxicity }(\mathrm{N}= \\
\text { 262) }\end{array}$ & $\begin{array}{l}P \\
\text { Value }\end{array}$ & $\mathrm{OR}(95 \% \mathrm{Cl})$ \\
\hline CT & 0.51 & 0.41 & - & 1(Reference) \\
\hline CC & 0.14 & 0.26 & 0.062 & $\begin{array}{l}0.46(0.20- \\
1.03)\end{array}$ \\
\hline TT & 0.05 & 0.22 & 0.006 & $\begin{array}{l}0.16(0.04- \\
0.59)\end{array}$ \\
\hline TC & 0.30 & 0.11 & 0.06 & $\begin{array}{l}1.96(0.98- \\
3.94)\end{array}$ \\
\hline $\begin{array}{l}\text { Haplotype } \\
\text { MDR1 }(1236 \mathrm{C} / \mathrm{T} \\
\text { and } 3435 \mathrm{C} / \mathrm{T})\end{array}$ & $\begin{array}{l}\text { Individuals with } \\
\text { ARV drug induced } \\
\text { hepatotoxicity }(\mathrm{N}=\mathbf{6 8})\end{array}$ & $\begin{array}{l}\text { Healthy controls } \\
(\mathrm{N}=310)\end{array}$ & $\begin{array}{l}\text { P- } \\
\text { Value }\end{array}$ & $\mathrm{OR}(95 \% \mathrm{Cl})$ \\
\hline CT & 0.51 & 0.35 & - & 1(Reference) \\
\hline $\mathrm{CC}$ & 0.14 & 0.30 & 0.03 & $\begin{array}{l}0.34(0.12- \\
0.94)\end{array}$ \\
\hline TT & 0.05 & 0.22 & 0.003 & $\begin{array}{l}0.09(0.02- \\
0.44)\end{array}$ \\
\hline TC & 0.30 & 0.13 & 0.11 & $\begin{array}{l}1.94(0.87- \\
4.37)\end{array}$ \\
\hline $\begin{array}{l}\text { Haplotype } \\
\text { MDR1 }(1236 \mathrm{C} / \mathrm{T} \\
\text { and } 3435 \mathrm{C} / \mathrm{T})\end{array}$ & $\begin{array}{l}\text { Individuals those without } \\
\text { hepatotoxicity }(\mathrm{N}=262)\end{array}$ & $\begin{array}{l}\text { Healthy controls } \\
(\mathrm{N}=310)\end{array}$ & $\begin{array}{l}\text { P- } \\
\text { Value }\end{array}$ & $\mathrm{OR}(95 \% \mathrm{Cl})$ \\
\hline CT & 0.41 & 0.35 & - & 1(Reference) \\
\hline $\mathrm{CC}$ & 0.26 & 0.30 & 0.31 & $\begin{array}{l}0.77(0.46- \\
1.28)\end{array}$ \\
\hline TT & 0.22 & 0.22 & 0.31 & $\begin{array}{l}0.75(0.43- \\
1.31)\end{array}$ \\
\hline $\mathrm{TC}$ & 0.11 & 0.13 & 0.43 & $\begin{array}{l}0.77(0.40- \\
1.48)\end{array}$ \\
\hline \multicolumn{5}{|c|}{$\begin{array}{l}\mathrm{N} \text {, total number of allele in individuals with ARV drug induced hepatotoxicity }(68) \text {, those without } \\
\text { hepatotoxicity }(262) \text { and healthy controls }(310) \text {. OR and } 95 \% \text { Cls were derived from logistic regression } \\
\text { model comparing the haplotype CT with other haplotypes. Significant } P \text { values are shown in bold } \\
(P<0.05)\end{array}$} \\
\hline
\end{tabular}

\subsection{MDR1 polymorphisms and stages of HIV}


The incidence of MDR1 polymorphism among individuals in different stages of HIV infection and the healthy controls was also studied as outlined in Table 5. A reduced frequency of MDR1 1236TT genotype was found among individuals intermediate stage of HIV infection than healthy control population (24.2\% versus $41.94 \%, \mathrm{OR}=0.43, \mathrm{P}=0.09$ ). The incidence of $M D R 13435 \mathrm{CT}$ and $3435 \mathrm{TT}$ genotypes did not vary among the individuals in different stages of HIV infection and healthy population.

Table 5

Distribution of MDR1 (1236 C/T and $3435 \mathrm{C} / \mathrm{T}$ ) genotypes among individuals in different stages of HIV infection versus healhty controls

\begin{tabular}{|c|c|c|c|c|c|c|c|}
\hline \multirow{2}{*}{$\begin{array}{l}\text { Genotype } \\
\text { MDR1 } \\
(1236 \\
\mathrm{C} / \mathrm{T})\end{array}$} & \multirow{2}{*}{$\begin{array}{l}\text { Healthy } \\
\text { controls } \\
\mathrm{N}=155 \\
(\%)\end{array}$} & \multicolumn{2}{|c|}{$\begin{array}{l}\text { Early stage of HIV } \\
\text { infection }\end{array}$} & \multicolumn{2}{|c|}{$\begin{array}{l}\text { Intermediate stage of } \\
\text { HIV infection }\end{array}$} & \multicolumn{2}{|c|}{$\begin{array}{l}\text { Advanced stage of } \\
\text { HIV infection }\end{array}$} \\
\hline & & $\begin{array}{l}\mathrm{N}=19 \\
(\%)\end{array}$ & OR (P) & $\begin{array}{l}N=33 \\
(\%)\end{array}$ & $\mathrm{OR}(\mathrm{P})$ & $\begin{array}{l}N=79 \\
(\%)\end{array}$ & OR (P) \\
\hline $\mathrm{CC}$ & $\begin{array}{l}69 \\
(44.52 \%)\end{array}$ & $\begin{array}{l}7 \\
(36.8 \%)\end{array}$ & $\begin{array}{l}1 \\
\text { (Reference) }\end{array}$ & $\begin{array}{l}18 \\
(54.5 \%)\end{array}$ & $\begin{array}{l}1 \\
\text { (Reference) }\end{array}$ & $\begin{array}{l}34 \\
(43.0 \%)\end{array}$ & $\begin{array}{l}1 \\
\text { (Reference) }\end{array}$ \\
\hline CT & $\begin{array}{l}65 \\
(41.94 \%)\end{array}$ & $\begin{array}{l}10 \\
(52.6 \%)\end{array}$ & $1.67(0.36)$ & $\begin{array}{l}8 \\
(24.2 \%)\end{array}$ & $0.43(0.09)$ & $\begin{array}{l}38 \\
(48.1 \%)\end{array}$ & $1.03(0.93)$ \\
\hline TT & $\begin{array}{l}21 \\
(13.54 \%)\end{array}$ & $\begin{array}{l}2 \\
(10.5 \%)\end{array}$ & $0.77(0.77)$ & $\begin{array}{l}7 \\
(21.2 \%)\end{array}$ & $0.93(0.90)$ & $\begin{array}{l}7 \\
(8.9 \%)\end{array}$ & $0.58(0.32)$ \\
\hline \multirow{2}{*}{$\begin{array}{l}\text { Genotype } \\
\text { MDR1 } \\
(3435 \\
\text { C/T) }\end{array}$} & \multirow{2}{*}{$\begin{array}{l}\text { Healthy } \\
\text { controls } \\
\mathrm{N}= \\
155(\%)\end{array}$} & \multicolumn{2}{|c|}{ Early stage of HIV } & \multicolumn{2}{|c|}{$\begin{array}{l}\text { Intermediate stage of } \\
\text { HIV }\end{array}$} & \multicolumn{2}{|c|}{$\begin{array}{l}\text { Advanced stage of } \\
\text { HIV }\end{array}$} \\
\hline & & $\begin{array}{l}N=19 \\
(\%)\end{array}$ & $\mathrm{OR}(\mathrm{P})$ & $\begin{array}{l}N=33 \\
(\%)\end{array}$ & OR (P) & $\begin{array}{l}N=79 \\
(\%)\end{array}$ & OR (P) \\
\hline $\mathrm{CC}$ & $\begin{array}{l}34 \\
(21.94 \%)\end{array}$ & $\begin{array}{l}4 \\
(21.1 \%)\end{array}$ & $\begin{array}{l}1 \\
\text { (Reference) }\end{array}$ & $\begin{array}{l}5 \\
(15.2 \%)\end{array}$ & $\begin{array}{l}1 \\
\text { (Reference) }\end{array}$ & $\begin{array}{l}15 \\
(19.0 \%)\end{array}$ & $\begin{array}{l}1 \\
\text { (Reference) }\end{array}$ \\
\hline CT & $\begin{array}{l}67 \\
(43.23 \%)\end{array}$ & $\begin{array}{l}6 \\
(31.6 \%)\end{array}$ & $0.41(0.23)$ & $\begin{array}{l}12 \\
(36.4 \%)\end{array}$ & $0.80(0.72)$ & $\begin{array}{l}32 \\
(40.5 \%)\end{array}$ & $0.83(0.66)$ \\
\hline TT & $\begin{array}{l}54 \\
(34.83 \%)\end{array}$ & $\begin{array}{l}9 \\
(47.4 \%)\end{array}$ & $0.93(0.92)$ & $\begin{array}{l}16 \\
(48.5 \%)\end{array}$ & $1.56(0.45)$ & $\begin{array}{l}32 \\
(40.5 \%)\end{array}$ & $1.11(0.81)$ \\
\hline
\end{tabular}

\subsection{Gene-environment interaction}

The distribution of MDR1 polymorphisms among ARV drug induced hepatotoxicity cases and those without hepatotoxicity and the healthy control group was analyzed by categorizing them on the basis of tobacco and alcohol consumption and NNRTI regimen as shown in Tables 6-9. The occurrence of polymorphisms of MDR1 (1236C/T and 3435C/T) was not different among the individual consuming tobacco in both the study populations and the healthy control group. MDR1 1236TT genotype was overrepresented among the tobacco consumers than the non-consumers in the ARV drug induced hepatotoxicity group (28.6\% versus $14.8 \%$ ) (Table-6). Also its occurrence was higher among the alcohol 
consumers than the non-consumers in the ARV drug induced hepatotoxicity group ( $28.6 \%$ versus $14.8 \%$, $\mathrm{OR}=1.50,95 \% \mathrm{Cl}: 0.13-17.35, \mathrm{P}=0.88)$. An increased incidence of 3435CT genotype of MDR1 was observed among the alcohol consumers than the nonusers in the individuals those without hepatotoxicity ( $50.0 \%$ versus $32.2 \%, \mathrm{OR}=2.47,95 \% \mathrm{Cl}: 0.79-7.70, \mathrm{P}=0.12$ ) (Table-7). The occurrence of genotype 1236TT of MDR1 was greater in nevirapine taking individuals those without hepatotoxicity than efavirenz users (14.1\% versus $8.7 \%, \mathrm{OR}=1.93,95 \% \mathrm{Cl}$ : $0.39-9.45, \mathrm{P}=0.42$ ). Also it was greater in individuals on nevirapine with ARV drug induced hepatotoxicity than efavirenz users $(21.7 \%$ versus $9.1 \%$, OR $=2.11,95 \% \mathrm{Cl}: 0.18$ $24.66, \mathrm{P}=0.55)$. This suggest that individuals with MDR11236TT genotype along with nevirapine usage may increase risk for severity of hepatotoxicity because of combined effect. The occurrence of MDR1 $1236 \mathrm{CT}$ and 1236TT genotypes were also higher in individuals those without hepatotoxicity on nevirapine than efavirenz users (43.7\% versus $33.3 \%, \mathrm{OR}=1.66,95 \% \mathrm{Cl}: 0.44-6.24, \mathrm{P}=0.45$ and $12.6 \%$ versus $8.3 \%, \mathrm{OR}=1.96,95 \% \mathrm{Cl}: 0.22-17.42, \mathrm{P}=0.55$ ) (Table-8). Among the ARV drug induced hepatotoxicity cases, who were on nevirapine and consumed alcohol, the dispersion of MDR1 1236TT genotype was greater when contrasted to the alcohol nonusers on nevirapine $(40.0 \%$ versus $16.67 \%$, OR= 2.21, 95\%Cl: $0.17-29.21, \mathrm{P}=0.55)$. This suggest that individuals with MDR1 1236TT genotype taking nevirapine and alcohol may increase the risk for severity of hepatotoxicity because of combined effect. The incidence of $3435 C T$ genotype of MDR1 was greater among the alcohol consumers on navirapine than alcohol non-consumers on navirapine, in individuals those without hepatotoxicity (44.74\% versus $33.33 \%, \mathrm{OR}=2.04,95 \% \mathrm{Cl}: 0.64-6.53, \mathrm{P}=0.23)$ (Table 9 ).

\subsection{Discussion}

We analyzed the association between MDR1 polymorphism and ARV drug induced hepatotoxicity. MDR1 encodes for the ATP- dependent membrane efflux transporter (14). P-glycoproteins are substrate for the genetic variant that impact patient drugs. The occurrence of MDR1 polymorphism changes from population to population [17]. We analyzed the MDR1 genotypes and haplotypes among the individuals with ARV drug induced hepatotoxicity and those without hepatotoxicity.

We found that the occurrence of MDR1 3435C/T polymorphism in our healthy control population is identical to the investigations done in European, North Indian, Turkish, and Asian populations [30-35] and contrasted with the similar studies done in the Chinese, Iranian and Thailand populations [27, 33, 36]. Also, the genotypic dispersal of MDR $11236 \mathrm{C} / \mathrm{T}$ polymorphism in our healthy individuals was almost alike to the similar investigations in the North Indian population [35]. However, it contrasted from the Mexican, Chinese and South African populations [18, 31, 38, 39]. We have done a genotype-phenotype analysis and found that the individuals with MDR11236TT genotype were at increased risk for severity of hepatotoxicity $(\mathrm{OR}=1.37, \mathrm{P}=0.57)$. However, due to the small number of ARV drug induced hepatotoxicity cases, the risk could not reach statistical significance. The low phenotypic expression was linked with polymorphisms in P-gp. People with 3435TT genotype were found to have lower levels of P-gp than CC and CT genotypes. Also, MDR1 3435C/T polymorphism was associated with the reduced risk of NNRTIinduced liver toxicity [25]. 
We also studied the gene-gene interactions to understand the synergistic impact of MDR1 polymorphism on ARV drug induced hepatotoxicity. The gene-gene interactions are known to have greater effects on gene expression than a single gene [40]. In our investigation, individuals possessing haplotype TC were more prone to a severe ARV drug induced hepatotoxicity ( $O R=1.96, P=0.06)$; and the ones with TT and CC haplotypes may have reduced risk for acquiring of $A R V$ drug induced hepatotoxicity $O R=0.16, P=0.006$; $\mathrm{OR}=0.46, \mathrm{P}=0.06 ; \mathrm{OR}=0.09, \mathrm{P}=0.003 ; \mathrm{OR}=0.34, \mathrm{P}=0.03$ ).

Likewise, we analyzed the association between the MDR1 genotypes and the stage of HIV infection. In our investigation, the incidence of MDR1 genotypes did not vary significantly among individuals of various stages of HIV infection as well as the healthy population. MDR1 1236CT, 1236TT, and 3435CT genotypes have been shown to be correlated with the HIV disease progression, haven't been found to regulate the susceptibility to HIV-1 infection [41]. Additionally, the patients with 3435 TT genotype had an increase in the CD4+ counts, following a treatment for half an year [23].

An analysis of the gene-environment interaction helps to know its impact on the disease etiology $[42,43]$. We did a case-only analysis to study the gene-environment interaction. We did not rather go for a casecontrol analysis in light of the fact that a case-control investigation, requires a coordination of cases with the control population [44]. HIV infected individuals who are naïve to ARV therapy and consume alcohol have been found to have a reduction in the CD4+ cell count [45]. Also, in the HIV infected women who consume tobacco, a diminished response to ARV therapy has been observed [46]. In our study, in ARV drug induced hepatotoxicity cases, who consume alcohol, MDR11236TT genotype exposed a risk for severe hepatotoxicity $(\mathrm{OR}=1.50, \mathrm{P}=0.88)$. Among the individuals those without hepatotoxicity and used alcohol, the incidence of 3435CT genotype posed a higher risk of acquisition of ARV drug induced hepatotoxicity $(\mathrm{OR}=2.47, \mathrm{P}=0.12)$ because of combined effect of gene and environment. Among the ARV drug induced hepatotoxicity cases on nevirapine, presence of MDR1 1236TT genotype was likely to be associated with the higher risk of severe hepatotoxicity $(O R=2.11, P=0.55)$. Whereas among individuals those without hepatotoxicity on nevirapine, MDR1 1236CT, 1236TT genotypes exposed a higher risk of acquisition of $A R V$ drug induced hepatotoxicity $(\mathrm{OR}=1.66, \mathrm{P}=0.45 ; \mathrm{OR}=1.96, \mathrm{P}=0.55)$. In individuals with ARV drug induced hepatotoxicity on nevirapine who consume, MDR1 1236TT genotype exposed a higher susceptibility to severe hepatotoxicity $(\mathrm{OR}=2.21, \mathrm{P}=0.55)$ because of combined effect of gene and environment.

In the individuals those without hepatotoxicity, who consume alcohol, MDR1 3435CT genotype showed a susceptibility to acquisition of $A R V$ drug induced hepatotoxicity $(O R=2.04, P=0.23)$. This suggests that individuals with MDR1 1236TT and 3435CT genotypes with or without ARV drug induced hepatotoxicity may have a combined effect on acquisition and severity of hepatotoxicity. Also, individuals on nevirapine, possessing 3435T allele has a reduced risk of hepatotoxicity [26]. Individuals with MDR1 1236T and $1235 \mathrm{~T}$ alleles have been associated with a diminished plasma NNRTI concentration, influencing the virological response to HAART [21]. Haas et al., (2005) have not found any significant association between $A B C B 1$ variations and plasma EFV concentrations [19]. 
The fact that this work can just assess the association and does not indicate causation, is one of the limiting points of the study. Also, the present investigation was planned to constitute a 1:4 proportion of cases versus controls but this couldn't be accomplished and we recruited them in 1:3 proportion which may be sufficient.

\section{Conclusions}

MDR1 haplotypes have an impact on the severity of ARV drug induced hepatotoxicity. In the individuals on nevirapine who consume alcohol, MDR1 1236TT and 3435CT genotypes, had a combined effect on acquisition and severity of hepatotoxicity.

MDR1 is associated with drug clearance. As MDR1 expression influences the response to NVP and EFV regimen. Therefore, further association studies between MDR1 polymorphism and plasma drug concentration would be done with a larger sample size. In addition, the association of polymorphisms of other drug transporter genes with plasma drug levels is required to comprehend the effect of genetic variants on treatment effect.

\section{Declarations}

Ethical Approval and Consent to participate: Study is approved from Institutional Ethics committee (IEC): NARI/EC/ICF version 1.0, dated 28 August 2013. Consent was taken from all the participants

Consent for publication: Written informed consent was obtained from the patient for publication of this report. A copy of the written consent is available for review by the Editor-in-Chief of this journal."

Availability of supporting data:The datasets used and/or analyzed during the current study are available from the corresponding author on reasonable request.

Competing interests: The authors declare that they have no competing interests in this section.

\section{Funding: Nil}

\section{Authors' contributions:}

HariOm Singh: Overall supervision

Dharmesh Samani: Experimental work

\section{Acknowledgements}

We greatly appreciate and acknowledge the clinic in-charges and other supporting staff of NARI for the recruitment of study participants. I would like to thank Kamini Jakhar for helping in editing the manuscript. 


\section{References}

1. O'Brien ME, Clark RA, Besch CL, Myers L, Kissinger P. (2003). Patterns and correlates of discontinuation of the initial HAART regimen in an urban outpatient cohort. $J$ Acquir Immune Defic Syndr, 34:407-14.

2. Van Dyke RB, Wang L, Williams PL, for the Pediatric ACTGCT. (2008). Toxicities Associated with Dual Nucleoside Reverse-Transcriptase Inhibitor Regimens in HIV-Infected Children. The Journal of Infectious Diseases, 198:1599-608.

3. Minzi OM, Irunde H, Moshiro C. (2009) .HIV patients presenting common adverse drug events caused by highly active antiretroviral therapy in Tanzania. Tanzan $J$ Health Res, 11:5-10.

4. Nagpal M, Tayal V, Kumar S, Gupta U. (2010). Adverse drug reactions to antiretroviral therapy in AIDS patients at a tertiary care hospital in India: A prospective observational study. Indian J Med Sci. 64:245-52.

5. Mascolini M. (2001). HIV news from Buenos Aires: Part 1-the Zahir and the band-aid. 1st IAS conference on HIV pathogenesis and treatment; July 8-11; Buenos Aires: APAC Mon, 274-89.

6. Croop JM. (1993) P-glycoprotein structure and evolutionary homologies. Cytotechnology, 12:1-32.

7. Jones PM, George AM. (2004). The ABC transporter structure and mechanism: perspectives on recent research. Cell Mol Life Sci.61:682-99.

8. Rosenberg MF, Callaghan R, Ford RC, Higgins CF. (1997). Structure of the multidrug resistance Pglycoprotein to $2.5 \mathrm{~nm}$ resolution determined by electron microscopy and image analysis. $J$ Biol Chem, 272:10685-94.

9. Cordon-Cardo C, O'Brien JP, Casals D, Rittman-Grauer L, Biedler JL, Melamed MR, Bertino JR. (1989) Multidrug-resistance gene (P-glycoprotein) is expressed by endothelial cells at blood-brain barrier sites. Proc Natl Acad Sci U S A, 86:695-8.

10. Pileri SA, Sabattini E, Falini B, Tazzari PL, Gherlinzoni F, Michieli MG, Damiani D, Zucchini L, Gobbi M, Tsuruo T, et al.(1991). Immunohistochemical detection of the multidrug transport protein P170 in human normal tissues and malignant lymphomas. Histopathology, 19:131-40.

11. Sakaeda T, Nakamura T, Okumura K. (2003). Pharmacogenetics of MDR1 and its impact on the pharmacokinetics and pharmacodynamics of drugs. 4:397-410.

12. Ambudkar SV, Dey S, Hrycyna CA, Ramachandra M, Pastan I, Gottesman MM. (1999) Biochemical, cellular, and pharmacological aspects of the multidrug transporter. Annu Rev Pharmacol Toxicol, 39:361-98.

13. Sugawara I, Kataoka I, Morishita Y, Hamada H, Tsuruo T, Itoyama S, Mori S. (1988) Tissue distribution of P-glycoprotein encoded by a multidrug-resistant gene as revealed by a monoclonal antibody, MRK 16. Cancer Res, 48:1926-9.

14. Thiebaut F, Tsuruo T, Hamada H, Gottesman MM, Pastan I, Willingham MC. (1987) Cellular localization of the multidrug-resistance gene product P-glycoprotein in normal human tissues. Proc Natl Acad Sci U S A 84:7735-8. 
15. Stormer E, von Moltke LL, Perloff MD, Greenblatt DJ.(2002). Differential modulation of Pglycoprotein expression and activity by non-nucleoside HIV-1 reverse transcriptase inhibitors in cell culture. Pharm Res, 19:1038-45.

16. Tozzi V. (2010). Pharmacogenetics of antiretrovirals. Antiviral Res, 85(1):190-200.

17. Chelule PK, Gordon M, Palanee T, Page T, Mosam A, Coovadia HM, Cassol S. (2003). MDR1 and CYP3A4 polymorphisms among African, Indian, and white populations in KwaZulu-Natal, South Africa. Clin Pharmacol Ther, 74:195-6.

18. Dong Q, Xu B, Tan Y, Liu Z, Tian L, Zhang B, Lin CK, Kung HF, Sung JJ, He ML. (2009) The genetic variability of MDR1 C3435T polymorphisms in four Southern Chinese populations. Biomed Pharmacother, 63:658-62.

19. Haas DW, Smeaton LM, Shafer RW, Robbins GK, Morse GD, Labbe L, Wilkinson GR, Clifford DB, D'Aquila RT, De Gruttola V, Pollard RB, Merigan TC, Hirsch MS, George AL Jr, Donahue JP, Kim RB. (2005). Pharmacogenetics of long-term responses to antiretroviral regimens containing Efavirenz and/or Nelfinavir: an Adult Aids Clinical Trials Group Study. J Infect Dis, 192:1931-42.j

20. Li YH, Wang YH, Li Y, Yang L. (2006) MDR1 gene polymorphisms and clinical relevance. Acta Genetica Sinica,33:93-104.

21. Salem AH, Fletcher CV, Brundage RC. (2014). Pharmacometric characterization of efavirenz developmental pharmacokinetics and pharmacogenetics in HIV-infected children. Antimicrob Agents Chemother, 58:136-43.

22. Zhu P, Zhu Q, Zhang Y, Ma X, Li Z, Li J, Chen J, Luo L, Ring HZ, Ring BZ, Su L. (2013) ABCB1 variation and treatment response in AIDS patients: initial results of the Henan cohort. PLoS One.8(1):e55197

23. Fellay J, Marzolini C, Meaden ER, Back DJ, Buclin T, Chave JP, Decosterd LA, Furrer H, Opravil M, Pantaleo G, Retelska D, Ruiz L, Schinkel AH, Vernazza P, Eap CB, Telenti A;Swiss HIV Cohort Study. (2002). Response to antiretroviral treatment in HIV-1-infected individuals with allelic variants of the multidrug resistance transporter 1: a pharmacogenetics study. Lancet, 359:30-6.

24. Leschziner GD, Andrew T, Pirmohamed M, Johnson MR. (2007). ABCB1 genotype and PGP expression, function and therapeutic drug response: a critical review and recommendations for future research. Pharmacogenomics J.7:154-79.

25. Ritchie MD, Haas DW, Motsinger AA, Donahue JP, Erdem H, Raffanti S, Rebeiro P, George AL, Kim RB, Haines JL, Sterling TR. (2006) Drug transporter and metabolizing enzyme gene variants and nonnucleoside reverse-transcriptase inhibitor hepatotoxicity. Clin Infect Dis, 43:779-82.

26. Haas DW, Bartlett JA, Andersen JW, Sanne I, Wilkinson GR, Hinkle J, Rousseau F, Ingram CD, Shaw A, Lederman MM, Kim RB; Adult AIDS Clinical Trials Group. (2006) Pharmacogenetics of nevirapineassociated hepatotoxicity: an Adult AIDS Clinical Trials Group collaboration. Clin Infect Dis, 43:783-6.

27. Pongstaporn W, Pakakasama S, Chaksangchaichote P, Pongtheerat T, Hongeng S, Permitr S. (2015). MDR1 C3435T and C1236T polymorphisms: association with high-risk childhood acute lymphoblastic leukemia. Asian Pac J Cancer Prev, 16:2839-43. 
28. Sole X, Guino E, Valls J, Iniesta R, Moreno V. (2006). SNPStats: a web tool for the analysis of association studies. Bioinformatics, 22:1928-9.

29. Cox A, Camp NJ, Nicklin MJ, di Giovine FS, Duff GW. (1998). An analysis of linkage disequilibrium in the interleukin-1 gene cluster, using a novel grouping method for multiallelic markers. Am J Hum Genet, 62:1180-8

30. Ghodke Y, Chopra A, Shintre P, Puranik A, Joshi K, Patwardhan B. (2011). Profiling single nucleotide polymorphisms (SNPs) across intracellular folate metabolic pathway in healthy Indians. Indian $J$ Med Res, 133:274-9.

31. Lakhan R, Misra UK, Kalita J, Pradhan S, Gogtay NJ, Singh MK, Mittal B. (2009). No association of ABCB1 polymorphisms with drug-refractory epilepsy in a north Indian population. Epilepsy Behav, 14:78-82.

32. Rubis B, Holysz H, Barczak W, Gryczka R, Lacinski M, Jagielski P, et al. (2012). Study of ABCB1 polymorphism frequency in breast cancer patients from Poland. Pharmacol Rep, 64(6):1560-6.

33. Shi NJ, Zhang WX, Zhang N, Zhong LN, Wang LP. (2017). Correlation of MDR1 gene polymorphisms with anesthetic effect of sevoflurane-remifentanil following pediatric tonsillectomy. Medicine (Baltimore), 96:e7002.

34. Tan EK, Chan DK, Ng PW, Woo J, Teo YY, Tang K, et al. (2005). Effect of MDR1 haplotype on risk of Parkinson disease. Arch Neurol, 62(3):460-4.

35. Rustemoglu A, Gumus-Akay G, Yigit S, Tasliyurt T. (2011). Analysis of common MDR1 (ABCB1) gene C1236T and C3435T polymorphisms in Turkish patients with familial Mediterranean fever. Genet Mol Res.10:3411-20.

36. Saidijam M, Mahjub H, Shabab N, Yadegarazari R. (2015).Simultaneous analysis of multidrug resistance 1(MDR1) C3435T, G2677T/A, and C1236T genotypes in Hamadan City population, West of Iran. Iran Biomed J, 19:57-62.

37. Jafar T, Prasad N, Agarwal V, Mahdi A, Gupta A, Sharma RK, et al. (2011). MDR-1 gene polymorphisms in steroid-responsive versus steroid-resistant nephrotic syndrome in children. Nephrol Dial Transplant, 26:3968-74.

38. Gutierrez-Rubio SA, Quintero-Ramos A, Duran-Cardenas A, Franco-Topete RA, Castro-Cervantes JM, Oceguera-Villanueva A, et al. (2015). $1236 \mathrm{C} / \mathrm{T}$ and $3435 \mathrm{C} / \mathrm{T}$ polymorphisms of the ABCB1 gene in Mexican breast cancer patients. Genet Mol Res, 14:1250-9.

39. Masebe TM, Bessong PO, Nwobegahay J, Ndip RN, Meyer D. (2012). Prevalence of MDR1 C3435T and CYP2B6 G516T polymorphisms among HIV-1 infected South African patients. Dis Markers, 32:43-50.

40. Palmer LJ, Cardon LR. (2005). Shaking the tree: mapping complex disease genes with linkage disequilibrium. Lancet, 366:1223-34.

41. Bellusci CP, Rocco CA, Aulicino PC, Mecikovsky D, Bologna R, Sen L, et al. (2010). MDR1 3435T and 1236T alleles delay disease progression to pediatric AIDS but have no effect on HIV-1 vertical transmission. AIDS, 24:833-40. 
42. Deng Y, Newman B, Dunne MP, Silburn PA, Mellick GD.(2004). Case-only study of interactions between genetic polymorphisms of GSTM1, P1, T1 and Z1 and smoking in Parkinson's disease. Neurosci Lett. 366:326-31.

43. Hunter DJ, Hankinson SE, Hough H, Gertig DM, Garcia-Closas M, Spiegelman D, et al. (1997). A prospective study of NAT2 acetylation genotype, cigarette smoking, and risk of breastcancer. Carcinogenesis, 18:2127-32.

44. Greenland S. (1980) The effect of misclassification in the presence of covariates. Am J Epidemiol, 112:564-9.

45. Samet JH, Cheng DM, Libman H, Nunes DP, Alperen JK, Saitz R. (2007). Alcohol consumption and HIV disease progression. J Acquir Immune Defic Syndr.46:194-9.

46. Feldman JG, Minkoff H, Schneider MF, Gange SJ, Cohen M, Watts DH, et al. (2006). Association of cigarette smoking with HIV prognosis among women in the HAART era: a report from the women's interagency HIV study. Am J Public Health. 96(6):1060-5.

\section{Supplementary Files}

This is a list of supplementary files associated with this preprint. Click to download.

- groupimage1.png 